\title{
Galectin 1 in dermatology: current knowledge and perspectives
}

\author{
Efstathia Pasmatzi ${ }^{1 凶}$, Christina Papadionysiou ${ }^{1}$, Alexandra Monastirli ${ }^{1,2}$, George Badavanis² $^{2}$, Dionysios Tsambaos ${ }^{1,2}$
}

\begin{abstract}
Galectins are a family of soluble proteins that are widely distributed in nature and bind to a variety of glycoproteins and glycolipids bearing $\beta$-galactoside residues. They are involved in highly important processes at the molecular and cellular level in human cutaneous and extracutaneous tissues, and they exert biological effects of paramount importance through their interaction with cytoplasmic and nuclear proteins and the components of the cell surface and extracellular matrix. Galectin 1 (Gal 1), the first galectin isolated, is a noncovalent homodimeric protein with a $14 \mathrm{kDa}$ monomer that contains one carbohydrate-recognition domain (CRD) and preferentially recognizes galactose- $\beta 1-4-\mathrm{N}$-acetyl-glucosamine sequences on $\mathrm{N}$ - or $\mathrm{O}$-linked glycans. Gal 1 occurs intracellularly, extracellularly, and on the cell surface. In the last few years Gal 1 has emerged as a multifaceted protein that exerts a wide spectrum of regulatory effects in diverse normal and abnormal tissues and conditions, indicating a tremendous therapeutic potential. This review summarizes current knowledge on the expression of Gal 1 in normal and diseased human skin, its implications in the pathogenesis, diagnosis, and prognosis of cutaneous disorders, and the novel approach to the treatment of these disorders offered by the use of Gal 1 or its inhibitors/antagonists.
\end{abstract}

Keywords: galectins, galectin 1, epidermis

Received: 19 May 2018 | Returned for modification: 3 October 2018 | Accepted: 16 October 2018

\section{Introduction}

One hundred thirty years ago, a protein discovered in castor bean extracts, was found to be capable of agglutinating animal erythrocytes (1). Since then, a considerable number of other proteins with agglutinating capacity have been found in various seeds and animals that are specific for binding to different glycans. All these agglutinins were termed lectins, from the Latin word legere 'to select' (2).

Today, it is known that lectins are proteins or glycoproteins of plant or animal origin widely distributed in nature that specifically bind to carbohydrate molecules or to carbohydrate functional groups of glycolipids and glycoproteins present in cell surfaces and also intracellularly (3).

Galectins constitute a family of $\beta$-galactoside-binding lectins that possess one or two unique structures termed "conserved carbohydrate-recognition domains" (CRDs), by means of which they bind with diverse carbohydrate ligands $(4,5)$. Nineteen mammalian galectins (13 in humans) have so far been identified (6), most of which consist of one CRD with a highly conserved amino acid sequence and a $\beta$-sandwich structure (characterized by two opposing antiparallel $\beta$-sheets), whereas a few others contain two homologous CRDs separated by a linker of up to 70 amino acids (4). Based on their structural properties, galectins can be classified into three major subfamilies:

1. The prototype, the largest subfamily, including galectins 1, 2, 5, $7,10,11,13,14$, and 15, which contain one CRD;

2. The tandem-repeat type, including galectins 4, 6, 8, 9, and 12, which contain two distinct CRDs in tandem connected by a linker; and

3. The chimera type, including galectin 3, which consists of unusual tandem repeats of proline- and glycine-rich short stretches fused onto the $\operatorname{CRD}(5,7,8)$.

Galectins recognize $\beta$-galactose; however, the binding affinity of galectin subfamilies differs depending on the structure of gly- coconjugates and the modifications of galactose residues, such as sialylation, fucosylation, and sulfation (5). Galectins are expressed both intracellularly and extracellularly, contain no classical signal sequence or transmembrane domain, and are secreted from the cells via nonclassical pathways (9). Galectins occur in various human cell types and tissues and in diverse mammals, fungi, nematodes, sponges, insects, and viruses. Many galectins are widely distributed in tissues, but few of them reveal a high tissue-specificity. Accumulating evidence suggests that galectins are involved in a wide variety of important molecular and cellular processes in both cutaneous and extracutaneous tissues.

This article summarizes current knowledge on the expression of galectin 1 (Gal 1) in normal and diseased human skin and on its potential functions and implications in the pathogenesis, diagnosis, prognosis, and treatment of cutaneous disorders. The overview of the available data is based on the results of an electronic literature research that was conducted on the Medline and Scopus databases through April 2018 using various combinations of the primary keyword galectins with relevant terms, the most important of which were keratinocytes, Langerhans cells, Merkel cells, melanocytes, lymphocytes, macrophages, skin, human adult epidermis, human embryonic epidermis, keratinization, infection, inflammation, immune response, cutaneous angiogenesis, melanoma, cutaneous neoplasms, basal cell carcinoma, squamous cell carcinoma, keratoacanthoma, actinic keratosis, xanthoma, nevi, Bowen's disease, tumor invasion, and metastasis.

\section{Galectin 1}

Gal 1, the first identified and best-studied prototypical member of the galectin family, is encoded in humans by the LGALS1 gene, which is located on chromosome 22 (q12) (10). It is a noncovalent homodimeric protein with a $14 \mathrm{kDa}$ monomer that contains one CRD and preferentially recognizes galactose- $\beta 1-4$ $\mathrm{N}$-acetyl-glucosamine sequences on $\mathrm{N}$ - or O-linked glycans (11). 
Gal 1 occurs intracellularly (in the cytoplasm and the nucleus), extracellularly, and on the cell surface (11).

Gal 1 is primarily released from the cells of adipose tissue, but it is also secreted by various other cell types. The cell types involved in the secretion and release of Gal 1 include human/porcine keratinocytes, thymic epithelial cells, fibroblasts, 3T3 cells, T- and B-cells, macrophages, dendritic cells, Langerhans cells, cultured stromal cells of human bone marrow, endothelial cells, and ovary cells (11-13). Various Gal 1 ligands are found on lymphocytes (CD7, CD43, and CD45), on endothelial cells (CD13, CD36, ROBO4, and integrins), and in the extracellular matrix (fibronectin, integrins, laminin, ROBO4, and GM1) (14).

The most important biological properties and actions of this galectin include involvement in morphogenesis, angiogenesis, regulation of the cell cycle, proliferation and immune response, cell-cell and cell-matrix adhesion, apoptosis, inflammation, tumor invasion and metastasis $\left(10,11,15^{-19}\right)$, regulation of the innate and the adaptive immune response (20), promotion of the subsidence of autoimmune inflammation and suppression of allergeninduced inflammation and antibacterial immune response (21), contribution to the induction of B cells' regulatory function (22) and to the escape of tumor cells from immune surveillance (21), involvement in tumoral angiogenesis, hypoxia and metastasis (23), and the mechanisms of microglial modulation, polarization, and remyelination (24).

\section{Normal human skin}

Gal 1 has been detected by Western blotting in human embryonic skin protein extracts with increasing amounts from 10 weeks to 14 weeks (estimated gestational age, EGA). Immunohistochemistry revealed no reactivity in fetal epidermal cells of 11-week EGA embryos, whereas connective tissue cells and dermal extracellular matrix were weakly positive for Gal 1 (25). At a later stage of epidermal morphogenesis (14 weeks EGA), basal epidermal cells revealed expression of Gal 1, whereas cells from the upper epidermal layers and the developing follicular buds remained negative for this galectin (25). These findings could be interpreted in terms of possible Gal 1 involvement in the regulation of keratinocyte proliferation.

In adult human skin, expression of Gal 1 has been found in the cytoplasm of keratinocytes in all layers of normal epidermis (13, 26-28), as well as in hair follicles and in the extracellular matrix of the dermis $(26,29)$. Furthermore, Gal 1 has been found in both the nucleus and the cytoplasm of normal fibroblasts and Langerhans cells (13) and in the cytoplasm of human epidermal melanocytes (10).

Because Gal 1 stimulates the maturation and migration of human dendritic cells, it has been suggested that it may contribute to the initiation of cutaneous immune response $(30,31)$. It has been shown that this galectin is capable of mediating cell-matrix interactions that are of essential importance in cell migration, re-epithelization, and wound healing $(32,33)$. Moreover, Dvorankova et al. (34) reported that Gal 1 can induce both the conversion of dermal fibroblasts into myofibroblasts and the production of extracellular matrix.

\section{Cutaneous and systemic disorders}

\section{A. Psoriasis}

In contrast to the keratinocytes in all layers of normal human epidermis that express Gal 1, those of the lesional psoriatic epidermis reveal no cytoplasmic and/or nuclear immunoreactivity (27). It therefore seems reasonable to suggest that the lack of Gal 1 expression in psoriatic epidermis could be associated with the abnormal keratinization and/or increased proliferation of keratinocytes in psoriatic lesions. Interestingly, a downregulation of the expression of this galectin was found in Langerhans cells and dendritic cells derived from the skin lesions of psoriatic patients, as compared to those of healthy controls (35), whereas large amounts of Gal 1 were found in the extracellular matrix of psoriatic dermis (27).

\section{B. Atopic dermatitis}

Interactions between immune dysregulation, genetic predisposition, impaired skin barrier, and bacterial and environmental factors are thought to be involved in the complex pathogenesis of atopic dermatitis, which still remains obscure $(36,37)$. Better understanding of the pathogenic mechanisms would greatly contribute to the identification of molecules and pathways responsible for the development of atopic dermatitis, which could serve as novel targets for its treatment.

In view of the multifaceted immunoregulatory properties of Gal 1, Correa et al. (38) evaluated the possible therapeutic efficacy of intraperitoneally applied recombinant Gal 1 on an ovalbumininduced atopic dermatitis model in BALB/c mice. In Gal 1-treated mice they found that Gal 1 was as effective as dexamethasone in causing clinically evident improvement of skin lesions, reduction in local eotaxin and interferon-gamma levels, suppression of eosinophil and mast cell infiltration, decrease of interleukin 17 plasma levels, activation of signal-regulated kinase, and downregulation of endogenous Gal 1. These very interesting findings indicate that the use of Gal 1 may represent a novel and promising approach to the treatment of atopic dermatitis, which is presently far from satisfactory.

\section{Cutaneous neoplasms}

\section{A. Epithelial}

The expression of this galectin has been extensively studied only in squamous cell carcinomas (SCCs) of the oral cavity and of the head and neck. In the healthy oral mucosa tissue, all epithelial cells were devoid of Gal 1 immunostaining except those of the basal layer (39). A weak nuclear or cytoplasmic expression of Gal 1 was demonstrated in both the oral papilloma and the oral SCC, indicating that this galectin is not differentially expressed in benign and malign oral tissue (40). Strong Gal 1 immunostaining was detected in early-stage oral SCC, being primarily localized in stromal cells, including fibroblasts, plasma cells, and giant cells. In late-stage oral SCCs, negative staining of Gal 1 was detected in the well-differentiated intermediate layer of carcinoma cells.

Gal 1 immunoreactivity was exclusively found in the less-differentiated cells around carcinomatous clusters, as well as in stromal plasma cells and fibroblasts (39).

During the metastatic stage, the only significant immunoreactivity was found in carcinoma cells at the tumor invasion front (39). Based on their findings, these authors suggested that Gal 1 may represent a novel molecular target for the diagnosis and prognosis of oral SCCs, and that its inhibitors might be useful in the management of early-stage oral carcinogenesis. More recently 
Noda et al. (41) determined that Gal 1 expression in gingival SCC significantly correlates with the histological differentiation of tumor cells, the extent of apoptosis and T cell infiltration, lymph node metastasis, and overall survival rate. Based on their findings, these authors suggested that this galectin may be used as a clinicopathological prognostic marker for gingival SCC.

In a thorough study, Valach et al. (42) found that upregulation of Gal 1 expression in head and neck SCCs significantly correlates with a) the presence of cancer-associated stromal myofibroblasts and $b$ ) the activation of genes linked to poor prognosis factors of head and neck SCCs, such as upregulation of nuclear factor $\kappa$-light-chain enhancer of activated B cells (NF- $\kappa B$ ) and splicing downregulation. Noda et al. (43) reported a high sensitivity and specificity of Gal 1 immunoreactivity in the detection of neoplastic cells in tissue specimens and smears derived from oral SCCs, and they suggested that this galectin may be a useful immunocytochemical marker for oral SCCs.

Because Gal 1 is a hypoxia-regulated protein and a prognostic marker in head and neck SSC, Koonce et al. (44) investigated the antitumor potential of a low molecular weight and non-peptidic Gal 1 inhibitor (OTXoo8) in athymic nude mice inoculated with two different cell lines from human head and neck SCCs. They found that OTXoo8 induced tumor cell normalization and inhibited tumor growth without any apparent toxicity. In view of these promising results, they suggested that the clinical application of OTXoo8 or other Gal 1 inhibitors may represent a novel approach to the treatment of head and neck SCCs. Interestingly, this inhibitor is also capable of directly and indirectly affecting cell cycle and survival and angiogenesis (6). Moreover, its in vitro and in vivo efficacy has been proven in several studies either as monotherapy or in combination with other regimens (45-47).

\section{B. Melanomas}

Gal 1 is highly expressed in melanomas; however, its immunoreactivity in these tumors is not associated with the overall or disease-free survival of the patients (10). This galectin is secreted by melanoma cells, exerting distinct stimulatory effects on their migration and also on angiogenesis $(48,49)$. Furthermore, it protects melanoma cells from the cytotoxic effects of chemotherapy and radiotherapy, and it assists them in escaping from immune surveillance mechanisms through induction of apoptosis of tumor-specific activated T cells attacking the melanoma $(49,50)$. In view of these properties and actions of Gal 1, it can be suggested that this galectin may be used as a novel molecular target in the treatment of melanoma because the reduction of its expression or its deletion could result in the loss of the immune privilege of malignant cells and in a marked decrease in both the resistance of this tumor to chemotherapy and radiotherapy and its metastatic potential (49). This hypothesis is supported by the findings of two more recent studies.

First, Yazawa et al. (51) studied the expression, identity, and function of the ligands of Gal 1 in the progression of melanoma and found an abundance of Gal 1 ligands in primary and metastatic melanoma that is lacking in epidermal melanocytes of normal human skin or the apparently normal skin surrounding the melanoma and in benign nevi. Furthermore, they demonstrated that the melanoma cell adhesion molecule (MCAM), which is implicated in the development of the tumor, was a major Gal 1 ligand. Interestingly, when MCAM-silenced melanoma cells were grown in mice deficient in Gal 1, melanoma growth was markedly reduced.

Second, Wu et al. (52) reported that in a subgroup of melanoma patients treated with ipilimumab and bevacizumab there was an increase in the serum levels of Gal 1 that was related to a decrease in survival. Interestingly, a different subgroup of melanoma patients revealed an enhancement of humoral immune response to Gal 1 that was associated with a favorable clinical outcome. Thus, these authors suggested that the levels of circulating Gal 1 and Gal 1 antibodies may be of importance for the efficacy of combined ipilimumab and bevacizumab treatment of melanoma and may represent a potential biomarker for immune therapy for melanoma.

In view of all these findings and the immunosuppressive, proangiogenic, and tumorigenic potential of Gal 1 (53), it seems reasonable to assume that this galectin alone or combined with immune checkpoint blockade may represent a significant therapeutic target and that the use of Gal 1 inhibitors/antagonists may indeed open a new and promising approach to the treatment of melanoma.

\section{T cell lymphomas}

In the lesions of patients with patch and tumor stage mycosis fungoides (MF), Gal 1 immunoreactivity is found in both the dermis and the epidermis in close proximity to infiltrating lymphocytes and Sézary cells, which exhibit strong expression of this galectin on their surface, whereas keratinocytes are negative. In the dermis, expression of Gal 1 is observed in scattered fibroblasts, endothelial cells, and macrophages (54).

It is known that Gal 1 can induce caspase-independent apoptosis of $\mathrm{T}$ cells and consequently suppression of $\mathrm{T}$ cell immunity (55). However, the susceptibility of T cells to Gal 1-induced apoptosis requires the expression of specific glycoprotein receptors on their surface, such as $\mathrm{CD}_{7}$ (56), containing the specific oligosaccharides that are recognized by Gal 1 (54). Thus, loss of CD7 expression on the surface of Sézary cells and alteration of their glycosylation (characterized by the occurrence of sialylated core 1 O-glycans) most probably contribute to the resistance of these cells to a variety of apoptosis-inducing agents, including Gal 1, and to the poor prognosis of T cell lymphoma (54). Interestingly, Rappl et al. (57) showed that the resistance of CD7- Sézary cells to Gal 1-mediated apoptosis may not only represent a mechanism of their immune escape but could also explain their progressive accumulation in the skin, peripheral blood, and other tissues of patients with Sézary syndrome. It may be suggested, therefore, that genes regulating glycosylation may be used as molecular targets for the development of novel compounds for the treatment of cutaneous T cell lymphomas (CTCLs) through enhancement of the susceptibility of malignant cells to apoptosis.

Cedeno-Laurent (58) reported that clonal malignant T cells in patients with advanced-stage (3 or 4) CTCLs reveal a Th2 cytokine pattern and strong intracellular Gal 1 expression. Moreover, plasma Gal 1 levels were increased in patients with leukemic CTCLs (L-CTCLs) as compared to healthy controls, and conditioned supernatants from primary L-CTCLs cell cultures caused a marked impairment of normal T-cell proliferation and a downregulation of Th1 responses in a $\beta$-galactoside-dependent manner, leading to impaired antitumor responses and increased susceptibility to infection. Based on their findings, these authors suggested that neutralization of Gal 1 interactions with its ligands may represent an effective approach to the augmentation of antineoplastic immune response in patients with L-CTCLs. 


\section{Miscellaneous: wounds and scars}

Gal 1 is expressed in human mesenchymal stem cells, acts as an autocrine negative growth regulator of fibroblasts, and is capable of inducing marked extracellular matrix formation and transforming growth factor beta (TGF- $\beta$ )-independent conversion of fibroblasts into myofibroblasts (34).

In a comparative study using proteomic analysis, Ong et al. (59) found a significant increase of Gal 1 expression in keloid scars (KS) as compared to normal skin. In addition, Gai et al. (60), in an experimental study on skin wound healing using Sprague Dawley rats, found an increase in the expression of Gal 1 during the early phages of re-epithelialization followed by a significant decrease thereafter, indicating that this galectin may play a significant role in the early phases of wound healing and contraction.

In a recent experimental study, Lin et al. (61) investigated the role of this galectin in cutaneous wound healing using Gal 1 knockout mice. They found that Gal 1 induced the activation, migration, and proliferation of myofibroblasts, accelerated the healing of wounds, and decreased the mortality of diabetic animals with cutaneous wounds. Taken together, the findings of these studies suggest that Gal 1 could be used in the development of a novel approach to the prevention of and/or therapy for cutaneous wounds.

\section{Conclusion}

Galectins are a family of small and highly conserved lectins that are widely distributed in nature. They bind to a variety of glycoproteins and glycolipids bearing $\beta$-galactoside residues and interact with diverse non-glycosylated molecules within the nucleus and the cytoplasm.

Gal 1, the first identified and best-studied prototypical member of the galectin family, is involved in a wide variety of biological processes in cutaneous and extracutaneous tissues.

In normal adult human skin, Gal 1 is expressed in the cytoplasm of keratinocytes in all epidermal layers, hair follicles, the extracellular matrix of the dermis, normal fibroblasts, Langerhans cells, and human epidermal melanocytes. Abnormalities in their expression are associated with various cutaneous disorders and are thought to be involved in the pathogenic mechanisms of these disorders.

Recent studies have shown that Gal 1 may be regarded as a useful diagnostic and prognostic factor in oral SCCs and that its administration may represent a novel and effective approach for the treatment of atopic dermatitis and cutaneous wounds. Accumulating experimental and clinical evidence strongly suggests that selective inhibition of this galectin or its interactions with its own ligands may open up entirely new avenues in the treatment of cutaneous neoplastic disorders and may provide a more thorough understanding of and greater insight into the mechanisms of their pathogenesis and also into the processes underlying the biological function of Gal 1 (Fig. 1).

\section{Dedicated to the memory of Ourania Marselou-Kinti.}

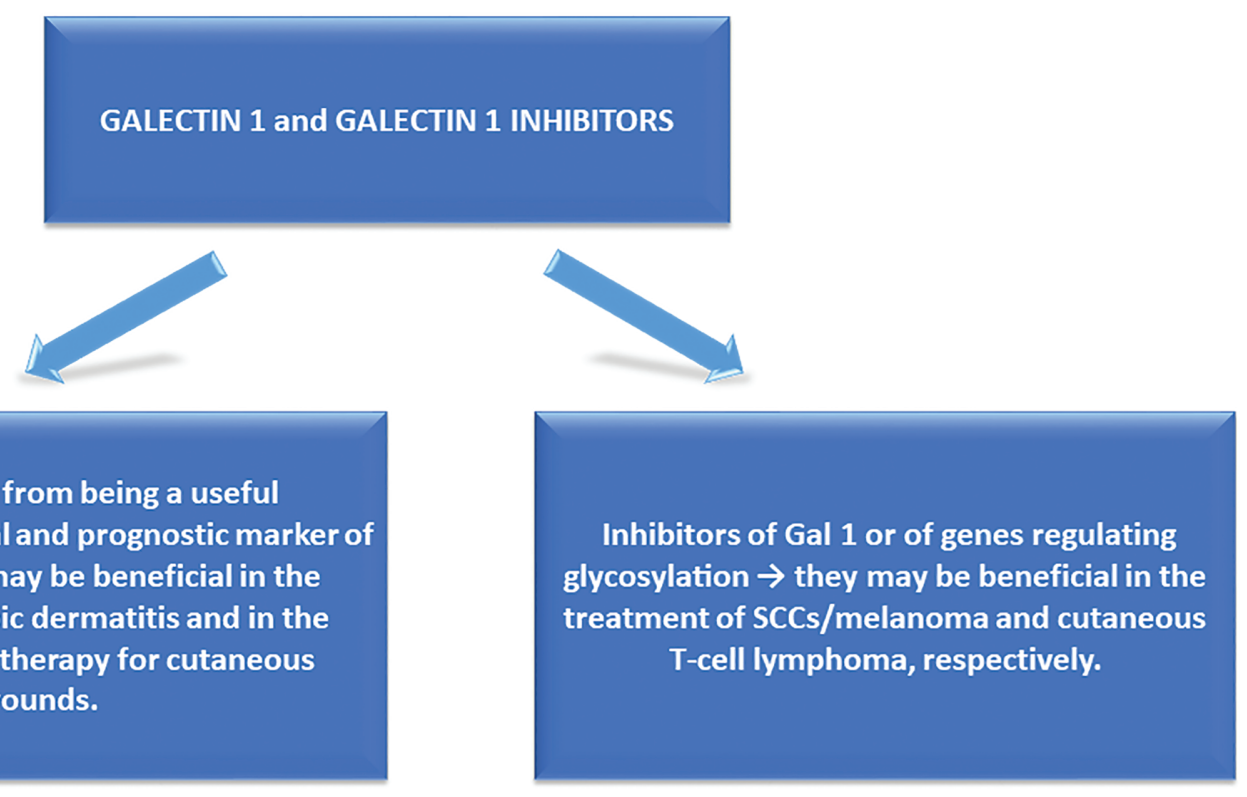

Figure 1 | Positive effects and potential therapeutic targets of Galectin 1 (Gal 1) and Gal 1 inhibitors. SCC = squamous cell carcinoma.

\section{References}

1. Stillmark H. Über Ricin, ein Giftiges Fragment aus den Samen von Ricinus comm. L. und einigen anderen Euphorbiaceen; Kaiserliche Universität zu Dorpat: University of Tartu; 1888.

2. Varki A, Cummings RD, Esko JD, Freeze HH, Stanley P, Bertozzi CR, Hart GW, Etzler ME, editors. Essentials of glycobiology. 2nd ed. Cold Spring Harbor, NY: Cold Spring Harbor Laboratory Press; 2009. Chapter 33.

3. Brooks SA. Lectin histochemistry: historical perspectives, state of the art, and the future. Methods Mol Biol. 2017;1560:93-107.

4. Larsen L, Chen HH, Saegusa J, Liu FT. Galectin-3 and the skin. J Dermatol Sci. 2011;64:85-91.
5. Kobayashi JN. Tissue- and cell-specific localization of galectins, b-galactosebinding animal lectins, and their potential functions in health and disease. Anat Sci Int. 2017;92:25-36.

6. Wdowiak K, Francuz T, Gallego-Colon E, Ruiz-Agamez N, Kubeczko M, Grochoła I, et al. Galectin targeted therapy in oncology: current knowledge and perspectives. Int J Mol Sci. 2018;10:19.

7. Yang RY, Rabinovich GA, Liu FT. Galectins: structure, function and therapeutic potential. Expert Rev Mol Med. 2008;13;10:e17.

8. Brauer R, Shoshan E, Kamiya T, Bar-Eli M. The sweet and bitter sides of galectins in melanoma progression. Pigment Cell Melanoma Res. 2012;25:592-601. 
9. Panjwani N. Role of galectins in re-epithelialization of wounds. Ann Transl Med. 2014;2:89-100.

10. Bolander A, Agnarsdottir M, Stromberg S, Ponten F, Hesselius P, Uhlen M, et al. The protein expression of TRP-1 and galectin-1 in cutaneous malignant melanomas. Cancer Genomics Proteomics. 2008;5:293-300.

11. Elola MT, Chiesa ME, Alberti AF, Mordoh J, Fink NE. Galectin-1 receptors in different cell types. J Biomed Sci. 2005;12:13-29.

12. Purkrabkova T, Smetana K Jr, Dvorankova B, Holikova Z, Böck C, Lensch M, et al. New aspects of galectin functionality in nuclei of cultured bone marrow stromal and epidermal cells: biotinylated galectins as tool to detect specific binding sites. Biol Cell. 2003;95:535-45.

13. Chen HY, Lo CH, Hsu D, Liu FT. Galectins and cutaneous immunity. Derm Sinica. 2012;30:121-7.

14. Dings RP, Kumar N, Miller MC, Loren M, Rangwala H, Hoye TR, et al. Structurebased optimization of angiostatic agent $6 \mathrm{DBF} 7$, an allosteric antagonist of galectin-1. J Pharmacol Exp Ther. 2013;344:589-99.

15. Kuwabara I, Timoshenko AV, Gorudko IV, Maslakova OV, André S, Liu FT, et al Analysis of selected blood and immune cell responses to carbohydrate-dependent surface binding of proto- and chimera-type galectins. Mol Cell Biochem. 2003;250:139-49.

16. Liu FT, Patterson RJ, Wang JL. Intracellular functions of galectins. Biochim Biophys Acta. 2002;1572:263-73.

17. Rabinovich GA, Rubinstein N, Toscano MA. Role of galectins in inflammatory and immunomodulatory processes. Biochim Biophys Acta. 2002;1572:274-84.

18. Chung CD, Patel VP, Moran M, Lewis LA, Miceli MC. Galectin-1 induces partia TCR zeta-chain phosphorylation and antagonizes processive TCR signal transduction. J Immunol. 2000;165:3722-9.

19. Moiseeva EP, Javed Q, Spring EL, de Bono DP. Galectin 1 is involved in vascular smooth muscle cellular proliferation. Cardiovasc Res. 2000;45:493-502.

20. Potikha T, Ella E, Cerliani JP, Mizrahi L, Pappo O, Rabinovich GA, et al. Galectin 1 is essential for efficient liver regeneration following hepatectomy. Oncotarget. 2016;7:31738-54

21. Sundblad V, Morosi LG, Geffner JR, Rabinovich GA. Galectin-1: A jack-of-all-trades in the resolution of acute and chronic inflammation. J Immunol. 2017;199:372130

22. Alhabbab R, Blair P, Smyth LA, Ratnasothy K, Peng Q, Moreau A, et al. Galectin-1 is required for the regulatory function of B cells. Sci Rep. 2018;8:2725.

23. Storti P, Marchica V, Airoldi I, Donofrio G, Fiorini E, Ferri V, et al. Galectin-1 suppression delineates a new strategy to inhibit myeloma-induced angiogenesis and tumoral growth in vivo. Leukemia. 2016;30:2351-63.

24. Rinaldi M, Thomas L, Mathieu P, Carabias P, Troncoso MF, Pasquini JM, et al. Galectin-1 circumvents lysolecithin-induced demyelination through the modulation of microglial polarization/phagocytosis and oligodendroglial differentiation. Neurobiol Dis. 2016;96:127-43.

25. Van de Brule F, Fernandez P, Buicu C, Liu FT, Jackers P, Lambotte R, et al. Differential expression of galectin-1 and galectin-3 during first trimester human embryogenesis. Dev Dyn. 1997;209:399-405.

26. Akimoto Y, Hirabayashi J, Kasai K, Hirano H. Expression of the endogenous 14kDa beta-galactoside-binding lectin galectin in normal human skin. Cell Tissue Res. 1995;280:1-10.

27. Lacina L, Plzakova Z, Smetana K, Stork J, Kaltner H, Andre S. Glycophenotype of psoriatic skin. Folia Biologica (Praha). 2006;52:10-15.

28. Cada Z, Smetana K Jr, Lacina L, Plzáková Z, Stork J, Kaltner H, et al. Immunohis tochemical fingerprinting of the network of seven adhesion/growth-regulatory lectins in human skin and detection of distinct tumour-associated alterations. Folia Biol (Praha). 2009;55:145-52.

29. Klima J, Smetana K Jr, Plzakova Z, Liu FT, Stork J, Kaltner H, et al. Comparative phenotypic characterization of keratinocytes originating from hair follicles. Mol Histol. 2005;36:89-96.

30. Fulcher JA, Hashimi ST, Levroney EL, Pang M, Gurney KB, Baum LG, et al. Galectin-1-matured human monocyte-derived dendritic cells have enhanced migration through extracellular matrix. J Immunol. 2006;177:216-26.

31. Fulcher JA, Chang MH, Wang S, Almazan T, Hashimi ST, Eriksson AU, et al. Galectin-1 co-clusters CD43/CD 45 on dendritic cells and induces cell activation and migration through Syk and protein kinase C signaling. J Biol Chem. 2009;284: 26860-70.

32. Woo HJ, Shaw LM, Messier JM, Mercurio AM. The major non-integrin laminin binding protein of macrophages is identical to carbohydrate binding protein 35 (Mac-2). J Biol Chem. 1990;265:7097-9.

33. Liu FT. Galectins: a new family of regulators of inflammation. Clin Immunol. 2000;97:79-88.

34. Dvořánková $B$, Szabo $P$, Lacina L, Gal P, Uhrova J, Zima T, et al. Human galectins induce conversion of dermal fibroblasts into myofibroblasts and production of extracellular matrix: potential application in tissue engineering and wound repair. Cells Tissues Organs. 2011;194:469-80.

35. de la Fuente H, Perez-Gala S, Bonay P, Cruz-Adalia A, Cibrian D, Sanchez-Cuellar $\mathrm{S}$, et al. Psoriasis in humans is associated with down-regulation of galectins in dendritic cells. J Pathol. 2012;228:193-203.

36. Dainichi T, Hanakawa S, Kabashima K. Classification of inflammatory skin diseases: a proposal based on the disorders of the three-layered defense systems, barrier, innate immunity and acquired immunity. J Dermatol Sci. 2014;76:81-9.
37. Rerknimitr P, Otsuka A, Nakashima C, Kabashima K. The etiopathogenesis of atopic dermatitis: barrier disruption, immunological derangement, and pruritus. Inflamm Regen. 2017;37:14.

38. Corrêa MP, Andrade FEC, Gimenes AD, Gil CD. Anti-inflammatory effect of galectin-1 in a murine model of atopic dermatitis. J Mol Med (Berl). 2017;95:1005-15

39. Chiang WF, Liu SY, Fang LY, Lin CN, Wu MH, Chen YC, et al. Overexpression of galectin-1 at the tumor invasion front is associated with poor prognosis in earlystage oral squamous cell carcinoma. Oral Oncol. 2008;44:325-34.

40. Hossaka TA, Ribeiro DA, Focchi G, André S, Fernandes M, Lopes Carapeto FC, et al. Expression of galectins 1, 3 and 9 in normal oral epithelium, oral squamous papilloma, and oral squamous cell carcinoma. Dent Res J (Isfahan). 2014;11: 508-12.

41. Noda Y, Kishino M, Sato S, Hirose K, Sakai M, Fukuda Y, et al. Galectin-1 expression is associated with tumour immunity and prognosis in gingival squamous cell carcinoma. J Clin Pathol. 2017;70:126-33.

42. Valach J, Fík Z, Strnad H, Chovanec M, Plzák J, Cada Z, et al. Smooth muscle actin-expressing stromal fibroblasts in head and neck squamous cell carcinoma: increased expression of galectin-1 and induction of poor prognosis factors. Int J Cancer. 2012;131:2499-508

43. Noda Y, Kondo Y, Sakai M, Sato S, Kishino M. Galectin-1 is a useful marker for detecting neoplastic squamous cells in oral cytology smears. Hum Pathol. 2016, 52:101-9.

44. Koonce NA, Griffin RJ, Dings RPM. Galectin-1 inhibitor OTX008 induces tumo vessel normalization and tumor growth inhibition in human head and neck squamous cell carcinoma models. Int J Mol Sci. 2017;9:18.

45. Dings RP, Van Laar ES, Loren M, Webber J, Zhang Y, Waters SJ, et al. Inhibiting tumor growth by targeting tumor vasculature with galectin-1 antagonist anginex conjugated to the cytotoxic acylfulvene, 6-hydroxylpropylacylfulvene. Bioconjug Chem. 2010;21:20-7.

46. Dings RP, Miller MC, Nesmelova I, Astorgues-Xerri L, Kumar N, Serova M, et al. Antitumor agent calixarene 0118 targets human galectin-1 as an allosteric inhibitor of carbohydrate binding. J Med Chem. 2012;55:5121-9.

47. Zucchetti M, Bonezzi K, Frapolli R, Sala F, Borsotti P, Zangarini M, et al. Pharmacokinetics and antineoplastic activity of galectin-1-targeting OTXoo8 in combination with sunitinib. Cancer Chemother Pharmacol. 2013;72:879-87.

48. Thijssen VL, Postel R, Brandwijk RJ, Dings RP, Nesmelova I, Satijn S, et al. Galectin-1 is essential in tumor angiogenesis and is a target for antiangiogenesis therapy. Proc Natl Acad Sci USA. 2006;103:15975-80.

49. Lefranc F, Mathieu V, Kiss R. Galectin-1 as an oncotarget in gliomas and melanomas. Oncotarget. 2011;2:892-3.

50. Rubinstein N, Alvarez M, Zwirner NW, Toscano MA, Ilarregui JM, Bravo A, et al. Targeted inhibition of galectin-1 gene expression in tumor cells results in heightened T cell-mediated rejection: a potential mechanism of tumor-immune privilege. Cancer Cell. 2004;5:241-51.

51. Yazawa E, Geddes-Swenney J, Cedeno-Laurent F, Walley K, Barthel S, Opperman $\mathrm{M}$, et al. Melanoma cell galectin-1 ligands functionally correlate with malignant potential. J Invest Dermatol. 2015;135:1849-62.

52. Wu MH, Chen YL, Lee KH, Chang CC, Cheng TM, Wu SY, et al. Glycosylation-dependent galectin-1 / neuropilin-1 interactions promote liver fibrosis through ac tivation of TGF- $\beta$ - and PDGF-like signals in hepatic stellate cells. Sci Rep. 2017;7: 11006.

53. Astorgues-Xerri L, Riveiro ME, Tijeras-Raballand A, Serova M, Rabinovich GA, Bieche I, et al. OTX008, a selective small-molecule inhibitor of galectin-1, downregulates cancer cell proliferation, invasion and tumour angiogenesis. Eur J Cancer. 2014;50:2463-77.

54. Roberts A, Amano M, Felten C, Galvan M, Sulur G, Pinter-Brown L, et al. Galectin1-mediated apoptosis in mycosis fungoides: the roles of $C_{7} 7$ and cell surface glycosylation. Modern Pathol. 2003;16:543-51.

55. Hahn HP, Pang M, He J, Hernandez JD, Yang RY, Li LY, et al. Galectin-1 induces nuclear translocation of endonuclease $\mathrm{G}$ in caspase- and cytochrome c-independent T cell death. Cell Death Differ. 2004;11:1277-86.

56. Perillo NL, Pace KE, Seilhamer JJ, Baum LG. Apoptosis of T cells mediated by galectin-1. Nature. 1995;14;378:736-9.

57. Rappl G, Abken H, Muche JM, Sterry W, Tilgen W, André S, et al. CD4+CD7- leukemic T cells from patients with Sézary syndrome are protected from galectin1-triggered T cell death. Leukemia. 2002;16:840-5.

58. Cedeno-Laurent F, Watanabe R, Teague J, Kupper T, Clark R, Dimitroff C. Galectin-1 inhibits the viability, proliferation and Th1 cytokine production of nonmalignant cells in patients with leukemic cutaneous T-cell lymphoma. Blood. 2012; 119:3534-8.

59. Ong CT, Khoo YT, Mukhopadhyay A, Masilamani J, Do DV, Lim IJ, et al. Comparative proteomic analysis between normal skin and keloid scar. $\mathrm{Br}$ J Dermatol. 2010;162:1302-15.

6o. Gai P, Vasilenko Th, Kostelníková M, Jakubco J, Kovác I, Sabol F, et al. Open wound healing in vivo: monitoring binding and presence of adhesion/growth regulatory galectins in rat skin during the course of complete re-epithelialization. Acta Histochem Cytochem. 2011;44:191-9.

61. Lin YT, Chen JS, Wu MH, Hsieh IS, Liang CH, Hsu CL, et al. Galectin-1 accelerates wound healing by regulating the neuropilin-1/Smad3/NOX 4 pathway and ROS production in myofibroblasts. Invest Dermatol, 2015:135:258-68. 